\title{
Experimental Evaluation of CT Number Changes in 320-Row CBCT Volume Scan for Proton Range Calculation
}

\author{
Ryuta Hirai ${ }^{1,2^{*}}$, Ryosuke Kohno ${ }^{3}$, Yu Kumazaki ${ }^{2}$, Tetsuo Akimoto ${ }^{4}$, \\ Hidetoshi Saitoh ${ }^{5}$, Shingo Kato ${ }^{1,2}$ \\ ${ }^{1}$ Department of Radiation Oncology, Saitama Medical University, Saitama, Japan \\ ${ }^{2}$ Department of Radiation Oncology, Saitama Medical University International Medical Center, Saitama, Japan \\ ${ }^{3}$ The University of Texas, MD Anderson Cancer Center, Houston, TX, USA \\ ${ }^{4}$ Division of Particle Therapy, National Cancer Center Hospital East, Chiba, Japan \\ ${ }^{5}$ Division of Radiological Sciences, Faculty of Health Sciences, Tokyo Metropolitan University, Tokyo, Japan \\ Email: ^rhirai@saitama-med.ac.jp
}

How to cite this paper: Hirai, R., Kohno, R., Kumazaki, Y., Akimoto, T., Saitoh, H. and Kato, S. (2018) Experimental Evaluation of CT Number Changes in 320-Row CBCT Volume Scan for Proton Range Calculation. International Journal of Medical Physics, Clinical Engineering and Radiation Oncology, 7, 141-150

https://doi.org/10.4236/ijmpcero.2018.72012

Received: February 26, 2018

Accepted: May 1, 2018

Published: May 4, 2018

Copyright $\odot 2018$ by authors and Scientific Research Publishing Inc. This work is licensed under the Creative Commons Attribution International License (CC BY 4.0).

http://creativecommons.org/licenses/by/4.0/

\begin{abstract}
We investigated the longitudinal positional dependence of CT number in 320-row Cone Beam Computed Tomography (CBCT) volume scan (320-row volume scan) using a simple geometric phantom (SGP) and a chest simulation phantom (CSP) in order to evaluate its effect on proton range calculation. The SGP consisted of lung substitute material (LSM) and a cylindrical phantom (CP) made of high-density polyethylene. The CSP was an anthropomorphic phantom similar to the human chest. The two phantoms were scanned using 320-row volume scan in various longitudinal positions from the central beam axis. In experiments using the SGP, an image blur at the boundary of the two materials became gradually evident when the LSM was placed far away from the beam central axis. The image blur of the phantom was consistent with the gradation in CT number. The maximum difference in CT numbers between the 64-row helical scan and 320-row volume scan at the boundary of the two materials was consistent with approximately $50 \%$ of the relative proton stopping power. In contrast, the CT number profile in each longitudinal position was fairly consistent and longitudinal positional dependence rarely occurred in the CSP experiments. Pass lengths of CT beams through areas with widely different electron densities were shorter, and thus did not significantly impact CT numbers. Based on findings from the CSP experiments, we considered 320-row volume scan to be feasible for proton range calculation in clinical settings, although the relatively large longitudinal positional dependence of CT number should be accounted for when doing so.
\end{abstract}




\section{Keywords}

320-Row CBCT Volume Scan, CT Number, Proton Range, Relative Stopping Power

\section{Introduction}

320-row Cone Beam Computed Tomography (CBCT) volume scan (320-row volume scan) has 320 detector rows in the longitudinal direction, which enables a scan with a width of $16 \mathrm{~cm}( \pm 8 \mathrm{~cm}$ from the beam central axis) at 0.275 s/rotation. This method offers high time-resolution, because temporal errors can be minimized. Diagnostically, 320-row volume scan has been used for various types of imaging such as coronary CT angiography and myocardial blood flow assessments [1] [2].

320-row volume scan may also be useful in the field of radiation therapy. Some studies have reported on $4 \mathrm{D}$ photon and proton treatment planning using helical or conventional 4DCT images [3] [4] [5]. In general, 16 to 64-row CT helical scan is used for photon/proton treatment planning, but 320-row volume scan is rarely used. Indeed, only a few studies have reported on the use of 320-row volume scan for motion assessment of a moving target in radiation therapy planning [6]. To the best of our knowledge, no report has used 320-row volume scan for dose calculations. 320-row volume scan has the potential to obtain more accurate 4DCT images than helical or conventional CT, and may be useful in $4 \mathrm{D}$ proton treatment planning.

However, since 320-row volume scan has certain characteristics that differ from helical and conventional scans, its effects on CT number must be examined prior to clinical use. For instance, the imaging range per rotation in 320-row volume scan is wider than that for helical or conventional 4DCT, because the detector rows increase longitudinally. As X-ray intensity on the cathode side differs from that on the anode side of the X-ray tube, changes in CT number in the longitudinal position may occur due to this heel effect [7]. In addition, the large cone angle of 320-row volume scan may affect the CT image, especially in areas distant from the beam central axis. These factors may cause inaccurate image reconstruction [8]. Therefore, a thorough evaluation of the feasibility of 320 -row volume scan for clinical use in proton treatment planning is warranted.

For proton beam therapy, changes in relative proton stopping power due to changes in CT number may affect proton range because CT number is converted to relative proton stopping power in order to calculate proton range, which is important for calculating proton dose [9] [10] [11] [12]. Therefore, in this study, we investigated the longitudinal positional dependence of CT number in 320 -row volume scan using two types of phantoms in order to evaluate its effects on proton range calculation. 


\section{Materials and Methods}

\subsection{Simple Geometric Phantom Experiment}

A simple geometric phantom (SGP) was prepared to compare CT number profiles for 320-row volume scan and 64-row helical scan as the reference. The inspiratory phase of the lung substitute material (LSM) (LN-300: Gammex, FL, USA) was inserted into a cylindrical phantom (CP) made of high-density polyethylene (Niporon Hard $^{\circledR}$ : Tosoh, Tokyo, Japan) (Figure 1). The longitudinal edge position of the LSM was changed from $3 \mathrm{~cm}$ to $7 \mathrm{~cm}$ superior to the beam central axis in 1-cm increments (Figure 2).

This study used CT equipment (Aquilion ONE ${ }^{\mathrm{TM}}$ : Toshiba Medical Systems, Tochigi, Japan) and scanned the phantom with two scan modes, including a volume scan using a 320-row detector and a helical scan using a 64-row detector by static scans. Scan conditions for both were $120 \mathrm{kV}, 500 \mathrm{~mA}, 0.5 \mathrm{~s}$ exposure time per rotation, and $0.5 \mathrm{~mm}$ slice thickness. Circular regions of interest (ROI) $(1 \mathrm{~cm}$ in diameter) were placed at the center of the phantom in all CT slices. CT number profiles of the ROI were measured longitudinally in each phantom position as mentioned above. Longitudinal positional dependence of CT numbers
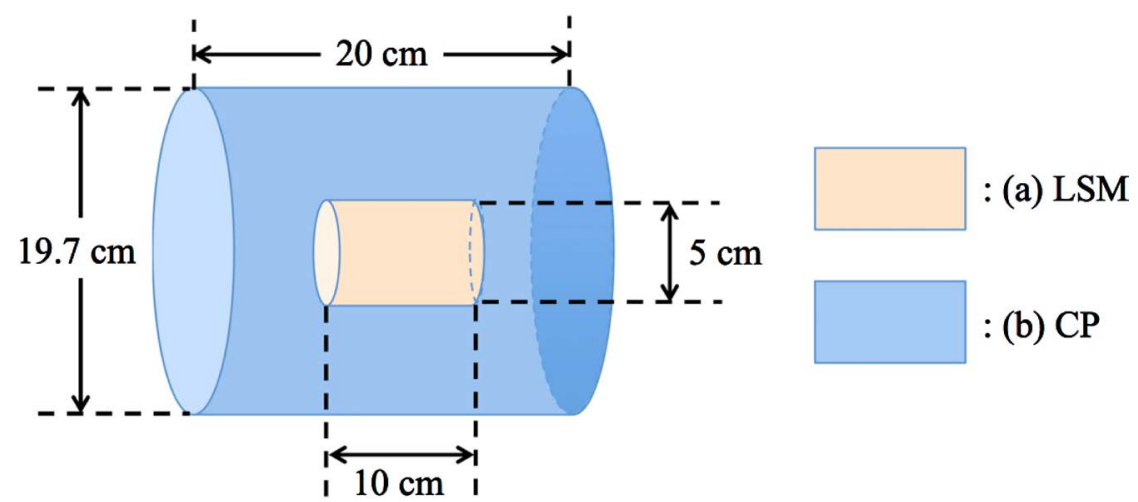

Figure 1. Schematic of the simple geometric phantom (SGP). The SGP consisted of lung substitute material (LSM) (a) and a cylindrical phantom (CP) (b). The LSM was a lung-simulated phantom with a diameter of $5 \mathrm{~cm}$ and length of $10 \mathrm{~cm}$, with a physical density of $0.3 \mathrm{~g} \mathrm{~cm}^{-3}$ and an electron density of $0.976 \times 10^{23} \mathrm{~cm}^{-3}$. The CP was a soft tissue-simulated phantom with a diameter of $19.7 \mathrm{~cm}$ and length of $20 \mathrm{~cm}$, with a physical density of approximately $0.95 \mathrm{~g} \mathrm{~cm}^{-3}$ and an electron density of approximately $3.3 \times 10^{23}$ $\mathrm{cm}^{-3}$.
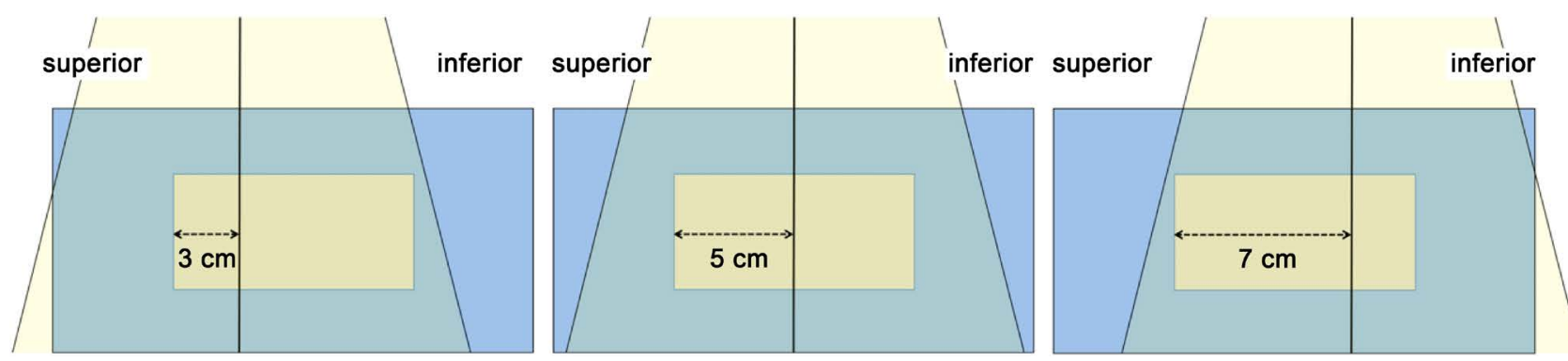

Figure 2. Geometric schematics of the phantom for CT scans in the edge position of the LSM at 3, 5, and $7 \mathrm{~cm}$ longitudinally from the beam central axis. 
was compared between 64-row helical scan and 320-row volume scan.

\subsection{Chest Simulation Phantom Experiment}

A chest simulation phantom (CSP) (Lung Man: Kyoto Kagaku, Kyoto, Japan) was used to compare CT number profiles of the two scan modes. The phantom was made of polyurethane for the soft tissue and epoxy for the skeleton (Figure 3) and measured $430 \mathrm{~mm}$ in width, $480 \mathrm{~mm}$ in height, and $940 \mathrm{~mm}$ in chest circumference. The simulated tumor ( $1 \mathrm{~cm}$ in diameter) (Tough Water: Kyoto Kagaku, Kyoto, Japan) was inserted in an arbitrary position of the CSP lung field.

The phantom was first scanned using 64-row helical scan to obtain reference data by static scan. Second, 320-row volume scan was performed with the beam central axis at the center of the simulated tumor. Following this, other volume scans were performed with the phantom position offset at $6 \mathrm{~cm}$ superior or inferior from the beam central axis. The ROI was placed in the slice at the center of the simulated tumor (Figure 4). CT number profiles of the ROI were measured, and differences in CT numbers were calculated for the helical scan versus volume scan of each position.

\section{Results}

\subsection{Simple Geometric Phantom Experiment}

Sagittal CT images of each scan condition are shown in Figure 5. The image blur at the boundary between the CP and the LSM became gradually evident, when the position of the $\mathrm{CP}$ moved far away from the beam central axis. A streak-like artifact was also observed at the edge of the LSM.

CT numbers of the ROI changed from approximately -80 Hounsfield Units (HU) to approximately $-750 \mathrm{HU}$, when the CT slice position was changed from the CP to the LSM. CT number profiles obtained at the edge of the LSM are shown in Figure 6. The image blur of the phantom was consistent with the

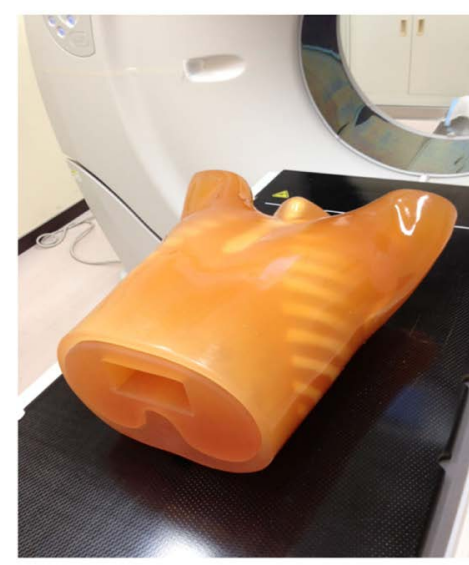

(a)

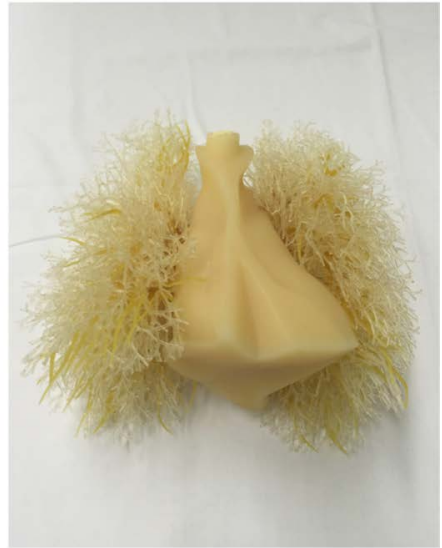

(b)

Figure 3. External views of the chest simulation phantom (CSP) (a) and its inner components (b). The CSP contained bony structure, soft tissue, heart, vessels, and lungs. The inner components are easily detachable, allowing for insertion of the simulated tumor. 


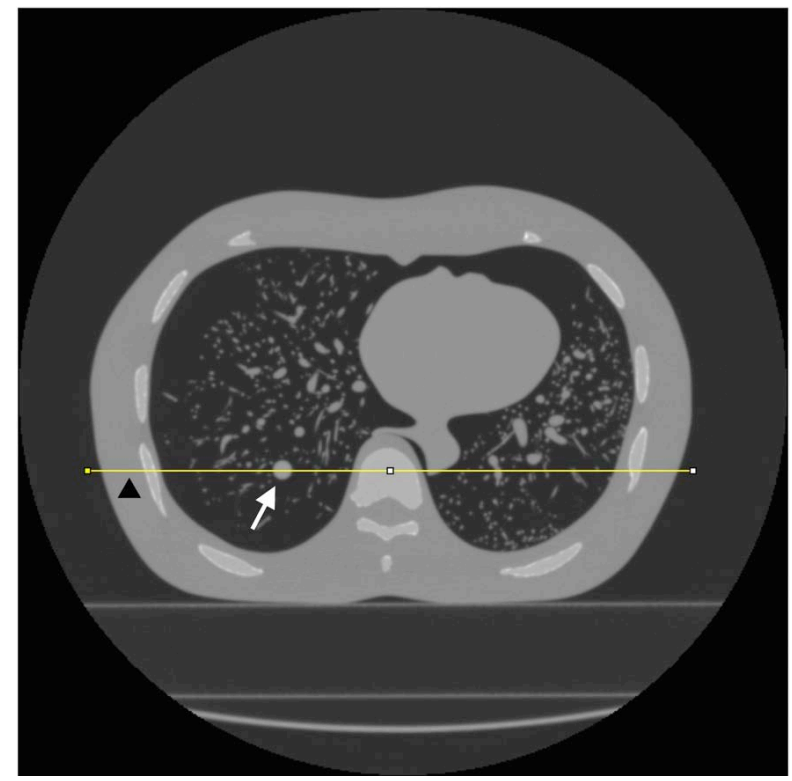

Figure 4. Placement of the ROI in the CT image to measure CT number profiles. CT image of the CSP with the simulated tumor (white arrow). The ROI was placed in the slice at the center of the simulated tumor (arrowhead) and CT profiles were obtained.

\section{4-row helical scan}

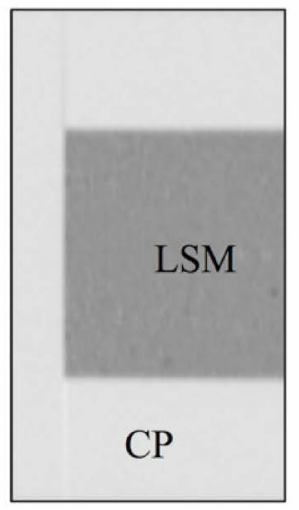

320-row volume scan

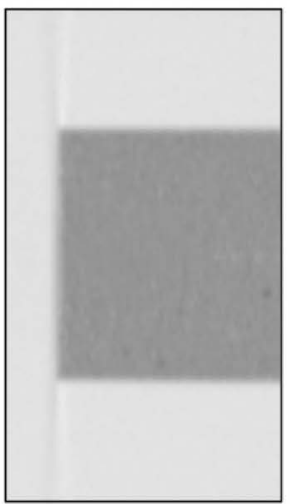

$3 \mathrm{~cm}^{*}$

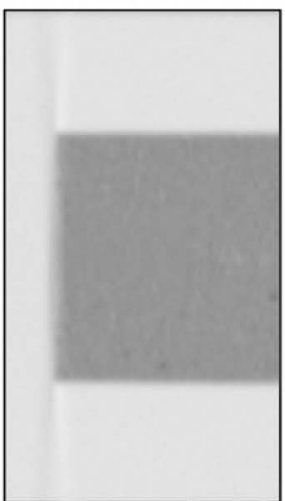

$4 \mathrm{~cm}^{*}$

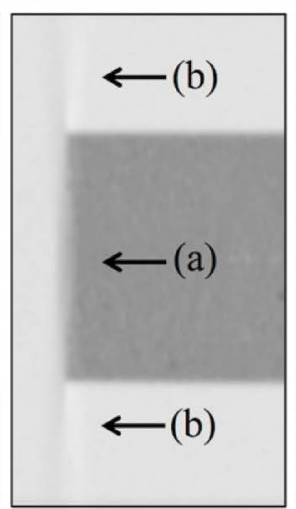

$5 \mathrm{~cm}^{*}$

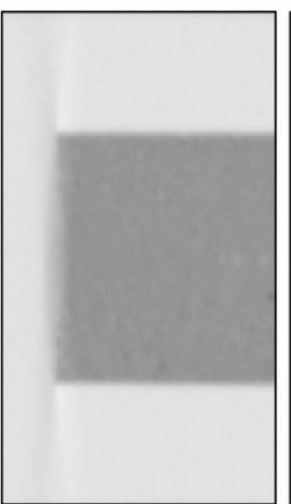

$6 \mathrm{~cm}^{*}$

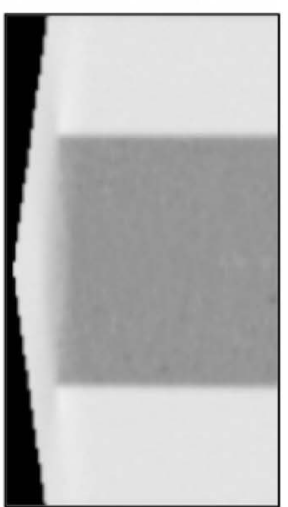

$7 \mathrm{~cm}^{*}$

Figure 5. Sagittal views of the SGP near the edge of the LSM obtained by 64-row helical scan and 320-row volume scan. *: CT images of 320-row volume scan were obtained from $3 \mathrm{~cm}$ to $7 \mathrm{~cm}$ from the beam central axis. The image blur (a) and the streak-like artifact (b) were gradually evident from $3 \mathrm{~cm}$ to $7 \mathrm{~cm}$. 


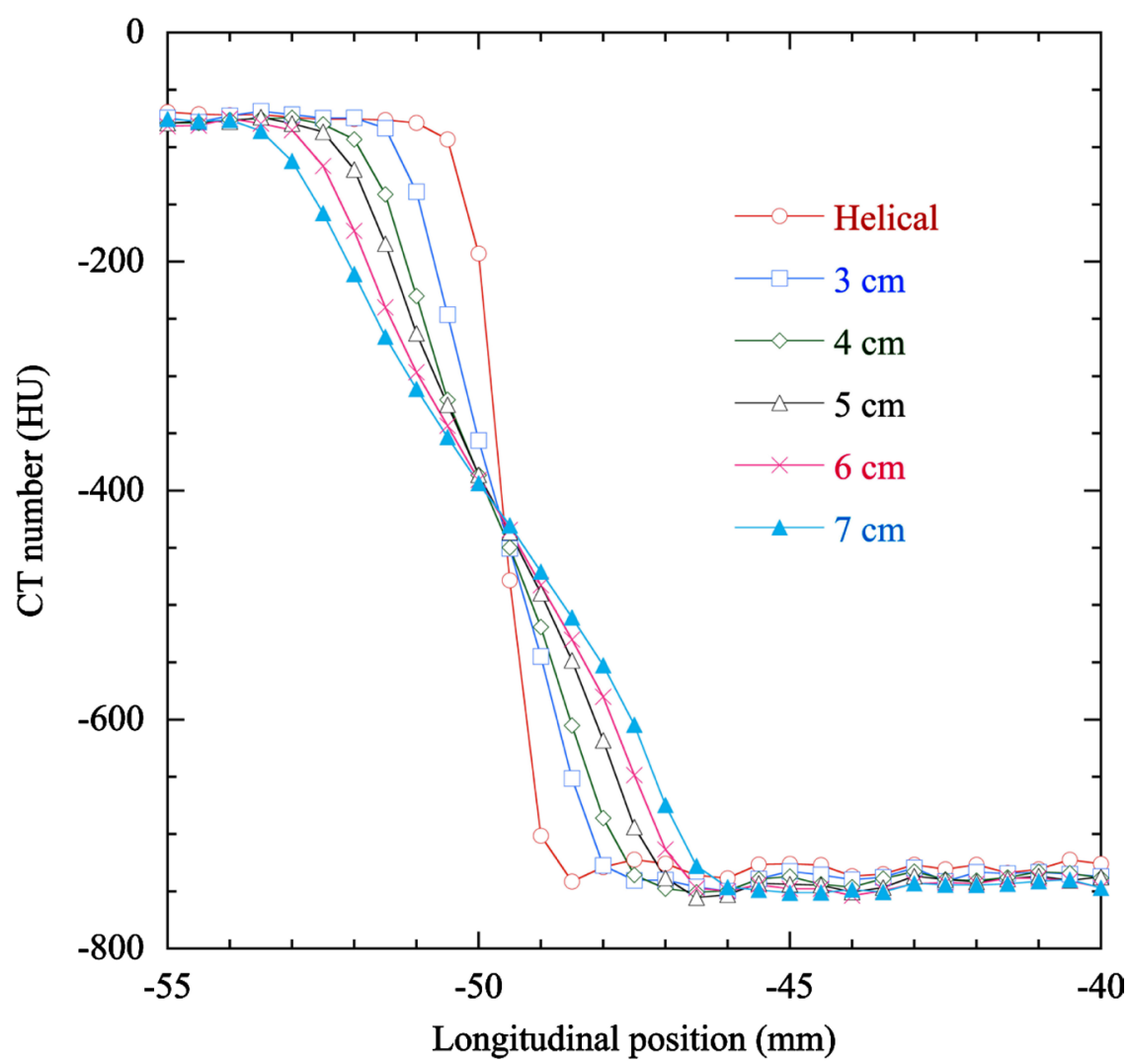

Figure 6. CT number profiles in the SGP. X-axis indicates the longitudinal edge position of the LSM from the beam central axis.

gradual change in CT number, and the blur length was approximately $3.5 \mathrm{~mm}$ in 320-row volume scan, when the edge of the LSM was placed $3 \mathrm{~cm}$ superior from the beam central axis. When the edge positions were $4 \mathrm{~cm}, 5 \mathrm{~cm}, 6 \mathrm{~cm}$, and $7 \mathrm{~cm}$ from the beam center, the corresponding blur lengths were $5 \mathrm{~mm}, 5.5 \mathrm{~mm}, 6$ $\mathrm{mm}$, and $7 \mathrm{~mm}$, respectively. Maximum differences in CT numbers between 64-row helical scan and 320-row volume scan at each phantom position are presented in Table 1 . These differences ranged from approximately $160 \mathrm{HU}$ to approximately $260 \mathrm{HU}$.

\subsection{Chest Simulation Phantom Experiment}

CT number profiles obtained using either 64-row helical scan or 320-row volume scan were fairly consistent across all conditions (Figure 7). Mean values for the differences in CT numbers ranged from 2.5 - 5.2 HU. The number of voxels in which the differences in CT numbers exceeded $100 \mathrm{HU}$ was 16 voxels/364 voxels (4.4\%) in the profile.

\section{Discussion}

In this study, we investigated the longitudinal positional dependence of CT number in 320-row volume scan using two phantoms. In experiments using the SGP, the image blur at the boundary of the two materials became gradually 
Table 1. Maximum differences in CT numbers between 64-row helical scan and 320-row volume scan $(\mathrm{HU})$.

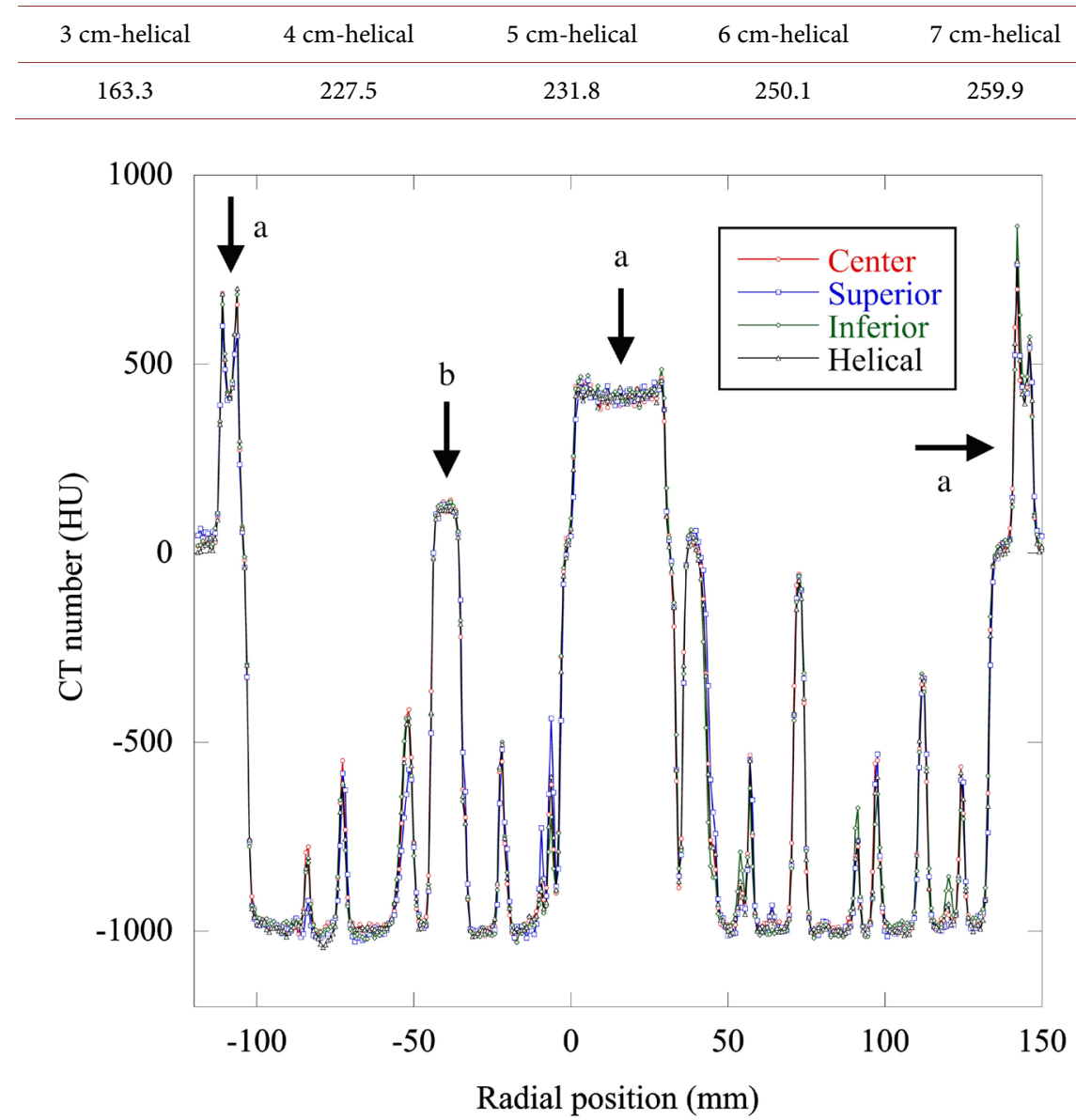

Figure 7. CT number profiles of the CSP. Peak areas of the profiles corresponded to bony structures (a) and the simulated tumor (b).

evident, when the position of the LSM was placed far away from the beam central axis with 320-row volume scan. The image blur of the phantom was consistent with the gradation in CT number (Figure 6).

The image blur can be explained as the partial volume effect of CT imaging. Generally speaking, the cone angle of 320-row CBCT is larger than that of conventional CT equipment. The incident beam angle of 320-row volume scan ranged from approximately $2.9-6.7$ degrees, when the edge of the LSM was placed $3-7 \mathrm{~cm}$ longitudinally from the beam center. Oblique beams coming in through the LSM and the CP could produce the partial volume effect between the two materials of different electron densities. As a result, we consider that changes in CT number at the boundary of the two materials could not be precisely expressed. The maximum width of the image blur was $7 \mathrm{~mm}$ and the maximum difference in CT numbers between 64-row helical scan and 320-row volume scan was approximately $260 \mathrm{HU}$ at the boundary of the two materials (Figure 6, Table 1). We converted CT number to relative proton stopping power for $235 \mathrm{MeV}$ proton beams using the polybinary calibration method based on 
the stoichiometric calibration method [9] [10] [11]. This difference is consistent with approximately $50 \%$ of the relative proton stopping power for this condition, which could affect proton range. In addition, the image blur and streak-like artifact may affect delineation of the target volume and organs at risk in treatment planning.

In experiments using the CSP, CT number profiles obtained using either 64-row helical scan or 320-row volume scan were fairly consistent across all conditions (Figure 7). CT numbers of the two scan modes were fairly comparable even when 320-row volume scans were performed with the phantom position offset at $6 \mathrm{~cm}$ superior or inferior to the beam central axis. The CSP had relatively fine geometry, and we observed shorter pass lengths for the CT beam through areas with widely different electron densities. For this reason, we consider the partial volume effect to not have significantly impacted CT numbers in 320 -row volume scan. However, the component placed in the phantom was relatively small. When a larger component is used, the pass length of the CT beam may be longer, and the image blur could become more evident.

The number of voxels in which the differences in CT numbers was $>100 \mathrm{HU}$ comprised only $4.4 \%$, and the mean differences in CT numbers between the two scan modes ranged from $2.5-5.2 \mathrm{HU}$ in experiments using the CSP. The mean difference in CT number of $5.2 \mathrm{HU}$ is generally consistent with approximately $1.8 \%$ of the proton range for the inspiratory phase of the lung, $1.1 \%$ for the expiratory phase of the lung, $0.3 \%$ for water, and $0.1 \%$ for cortical bone for 235 $\mathrm{MeV}$ proton beams. When proton beams pass through $5 \mathrm{~cm}$ of the inspiratory phase of the lung as they approach the target, a range error of approximately 0.9 $\mathrm{mm}$ occurs. This uncertainty in proton range is considered negligible when compared with other factors that induce changes in proton range [13]-[19]. As a result, we consider the changes in CT number when using 320-row volume scan to minimally affect proton range. However, the relatively large longitudinal positional dependence of CT number should be considered.

This study has a limitation regarding proton dose calculations. Given the difficulty of proton dose measurements in a heterogeneous medium, we did not conduct a dosimetric study. Further investigation will be needed to achieve accurate proton dose measurements.

\section{Conclusion}

The uncertainty in proton range caused by the longitudinal positional dependence of CT number in 320-row volume scan is considered negligible. Our findings from experiments using an anthropomorphic phantom suggest that the influence of 320-row volume scan on proton range calculation in the clinical setting is minimal.

\section{Acknowledgements}

The authors thank Tomoaki Tamaki (Department of Radiation Oncology, 
School of Medicine, Fukushima Medical University) for his assistance.

\section{Funding}

This work was supported in part by a grant from Toshiba Medical Systems.

\section{References}

[1] Tomizawa, N., Kanno, S., Maeda, E., Akahane, M., Torigoe, R. and Ohtomo, K. (2014) Minimizing the Acquisition Phase in Coronary CT Angiography Using the Second Generation 320-Row CT. Japanese Journal of Radiology, 32, 391-396. https://doi.org/10.1007/s11604-014-0321-1

[2] Kikuchi, Y., Oyama-Manabe, N., Naya, M., Manabe, O., Tomiyama, Y., Sasaki, T., Katoh, C., Kudo, K., Tamaki, N. and Shirato, H. (2014) Quantification of Myocardial Blood Flow Using Dynamic 320-Row Multi-Detector CT as Compared with 15O-H2O PET. European Radiology, 24, 1547-1556.

https://doi.org/10.1007/s00330-014-3164-3

[3] Matsugi, K., Nakamura, M., Miyabe, Y., Yamauchi, C., Matsuo, Y., Mizowaki, T. and Hiraoka, M. (2013) Evaluation of 4D Dose to a Moving Target with Monte Carlo Dose Calculation in Stereotactic Body Radiotherapy for Lung Cancer. Radiological Physics and Technology, 6, 233-240. https://doi.org/10.1007/s12194-012-0193-y

[4] Li, H., Liu, W., Park, P., Matney, J., Liao, Z., Chang, J., Zhang, X., Li, Y. and Zhu, R.X. (2014) Evaluation of the Systematic Error in Using 3D Dose Calculation in Scanning Beam Proton Therapy for Lung Cancer. J Appl Clin Med Phys, 15, 4810. https://doi.org/10.1120/jacmp.v15i5.4810

[5] Liu, W., Schild, S.E., Chang, J.Y., Liao, Z., Chang, Y., Wen, Z., Shen, J., Stoker, J.B., Ding, X., Hu, Y., Sahoo, N., Herman, M.G., Vargas, C., Keole, S., Wong, W. and Bues, M. (2016) Exploratory Study of 4D versus 3D Robust Optimization in Intensity Modulated Proton Therapy for Lung Cancer. International Journal of Radiation Oncology, Biology, Physics, 95, 523-533. https://doi.org/10.1016/j.ijrobp.2015.11.002

[6] Coolens, C., Bracken, J., Driscoll, B., Hope, A. and Jaffray, D. (2012) Dynamic Volume vs Respiratory Correlated 4DCT for Motion Assessment in Radiation Therapy Simulation. Medical Physics, 39, 2669-2681. https://doi.org/10.1118/1.4704498

[7] Mori, S., Endo, M., Nishizawa, K., Tsunoo, T., Aoyama, T., Fujiwara, H. and Murase, K. (2005) Enlarged Longitudinal Dose Profiles in Cone-Beam CT and the Need for Modified Dosimetry. Medical Physics, 32, 1061-1069.

https://doi.org/10.1118/1.1877852

[8] Coolens, C., Breen, S., Purdie, T.G., Owrangi, A., Publicover, J., Bartolac, S. and Jaffray, D.A. (2009) Implementation and Characterization of a 320-Slice Volumetric CT Scanner for Simulation in Radiation Oncology. Medical Physics, 36, 5120-5127. https://doi.org/10.1118/1.3246352

[9] Schneider, U., Pedroni, E. and Lomax, A. (1996) The Calibration of CT Hounsfield Units for Radiotherapy Treatment Planning. Physics in Medicine \& Biology, 41, 111-124. https://doi.org/10.1088/0031-9155/41/1/009

[10] Yohannes, I., Kolditz, D., Langner, O. and Kalender, W.A. (2012) A Formulation of Tissue- and Water-Equivalent Materials Using the Stoichiometric Analysis Method for CT-Number Calibration in Radiotherapy Treatment Planning. Physics in Medicine \& Biology, 57, 1173-1190. https://doi.org/10.1088/0031-9155/57/5/1173 
[11] Kanematsu, N., Matsufuji, N., Kohno, R., Minohara, S. and Kanai, T. (2003) A CT Calibration Method Based on the Polybinary Tissue Model for Radiotherapy Treatment Planning. Physics in Medicine \& Biology, 48, 1053-1064. https://doi.org/10.1088/0031-9155/48/8/307

[12] Inaniwa, T., Tashima, H. and Kanematsu, N. (2017) Optimum Size of a Calibration Phantom for x-Ray CT to Convert the Hounsfield Units to Stopping Power Ratios in Charged Particle Therapy Treatment Planning. Journal of Radiation Research, 1-9.

[13] Schuemann, J., Dowdell, S., Grassberger, C., Min, C.H. and Paganetti, H. (2014) Site-Specific Range Uncertainties Caused by Dose Calculation Algorithms for Proton Therapy. Physics in Medicine \& Biology, 59, 4007-4031. https://doi.org/10.1088/0031-9155/59/15/4007

[14] Chvetsov, A.V. and Paige, S.L. (2010) The Influence of CT Image Noise on Proton range Calculation in Radiotherapy Planning. Physics in Medicine \& Biology, 55, 141-149. https://doi.org/10.1088/0031-9155/55/6/N01

[15] Yang, M., Zhu, S.R., Park, P.C., Titt, U., Mohan, R., Virshup, G., Clayton, J. and Dong, L. (2012) Comprehensive Analysis of Proton Range Uncertainties Related to Patient Stopping-Power-Ratio Estimation using the Stoichiometric Calibration. Physics in Medicine \& Biology, 57, 4095-4115. https://doi.org/10.1088/0031-9155/57/13/4095

[16] Schaffner, B. and Pedroni, E. (1998) The Precision of Proton Range Calculations in Proton Radiotherapy Treatment Planning: Experimental Verification of the Relation between CT-HU and Proton Stopping Power. Physics in Medicine \& Biology, 43, 1579-1592. https://doi.org/10.1088/0031-9155/43/6/016

[17] Espana, S. and Paganetti, H. (2011) Uncertainties in Planned Dose Due to the Limited Voxel Size of the Planning CT When Treating Lung Tumors with Proton Therapy. Physics in Medicine \& Biology, 56, 3843-3856. https://doi.org/10.1088/0031-9155/56/13/007

[18] Kumazaki, Y., Akagi, T., Yanou, T., Suga, D., Hishikawa, Y. and Teshima, T. (2007) Determination of the Mean Excitation Energy of Water from Proton Beam Ranges. Radiation Measurements, 42, 1683-1691. https://doi.org/10.1016/j.radmeas.2007.10.019

[19] Kim, D.H., Cho, S., Jo, K., Shin, E., Hong, C.S., Han, Y., Suh, T.S., Lim, D.H. and Choi, D.H. (2018) Proton Range Verification in Inhomogeneous Tissue: Treatment Planning System vs. Measurement vs. Monte Carlo Simulation. PLoS ONE, 13, e0193904. https://doi.org/10.1371/journal.pone.0193904 